\section{PP-004 ANTIBIOTIC STEWARDSHIP PROGRAM; CONTEXTUAL ENVIRONMENT AND FRAMEWORK}

Naser Al-Khatib, Brenda Cholin, John Moraros. University of Saskatchewan

10.1136/jech-2013-203098.5

Introduction Antibiotic Stewardship is currently an important topic in Public Health, Hospital Epidemiology and Infectious Disease Epidemiology. Designing, implementing and evaluating an Antibiotic Stewardship Program (ASP) is mandates by accreditation agencies. Prairie North Regional Health Authority (PNRHA)- Saskatchewan assigned me to collect and analyse quantitatively and qualitatively - data related to antibiotic prescription habits of physicians in three hospitals and community clinics.

Objectives 1- to review the literature on antibiotic Stewardship and its programs 2- to understand the local antibiotics prescribing environment (using one 2010 data) 3- to design an Antibiotic Stewardship Program for the PNRHA

Methods Data collection by direct key stakeholders interviews, using hospital pharmacy, the lab and admission information systems. Community data were sourced from Saskatchewan Drug Plan administration - Using Excel and SPSS for data management, and biostatistics (parametric, non-parametric and variance analysis) - Using results for evidence based design of an Antibiotic Stewardship Program

Results The detailed descriptive statistics were very helpful to draw a baseline. These descriptive statistics shed the light on usage of 33 antibiotics in three different hospitals. These results were categorized based on specialty, main groups of antibiotics, per doctor, per antibiotic, per DOT (Duration of Therapy) and DOT per 1000 patient-day.

Conclusions There were recommendations on how to adopt the Antibiotic Stewardship and the suggested framework, as well as recommendations about data collection, analysis and measurements for future implementation of the Program. Specific steps were encouraged based on the exact results of specific antibiotics such as flouroquinolones, clindamycin, vancomycin and IV administration and conversion to oral antibiotics 